\{Preliminary version - accepted as a chapter in Aesthetics and Mathematics, a book under contract to Springer-Verlag.\}

\title{
Experiencing Meanings in Geometry ${ }^{1}$
}

By

\author{
David W. Henderson and Daina Taimina
}

\begin{abstract}
What geometrician or arithmetician could fail to take pleasure in the symmetries, correspondences and principles of order observed in visible things? Consider, even, the case of pictures: those seeing by the bodily sense the productions of the art of painting do not see the one thing in the one only way; they are deeply stirred by recognizing in the objects depicted to the eyes the presentation of what lies in the idea, and so are called to recollection of the truth - the very experience out of which Love rises.
\end{abstract}

— Plotinus (Greek philosopher-sage), The Enneads, II.9.16 [Plotinus] (204-270 AD)

In mathematics, as in any scientific research, we find two tendencies present. On the one hand, the tendency toward abstraction seeks to crystallize the logical relations inherent in the maze of material that is being studied, and to correlate the material in a systematic and orderly manner. On the other hand, the tendency toward intuitive understanding fosters a more immediate grasp of the objects one studies, a live rapport with them, so to speak, which stresses the concrete meaning of their relations.

As to geometry, in particular, the abstract tendency has here led to the magnificent systematic theories of Algebraic Geometry, of Riemannian Geometry, and of Topology; these theories make extensive use of abstract reasoning and symbolic calculation in the sense of algebra. Notwithstanding this, it is still as true today as it ever was that intuitive understanding plays a major role in geometry. And such concrete intuition is of great value not only for the research worker, but also for anyone who wishes to study and appreciate the results of research in geometry.

— David Hilbert (The "father of Formalism" in mathematics) [Hilbert], p. iii (1934)

It's a thing that nonmathematicians don't realize. Mathematics is actually an aesthetic subject almost entirely.

-- John H. Conway (Professor of Mathematics, Princeton), as quoted in [Spencer] (2001)

The artist and scientist both live within and play active roles in constructing human mental and physical landscapes. That they should share structural intuitions is less surprising than inevitable. What is surprising and wonderful is how these intuitions have manifested themselves in the works of innovative artists and scientists in culturally apposite ways.

-- Martin Kemp (Art historian) [Kemp], p.7 (2000)

These authors all stress the importance of the deep experience of meanings. It is these experiences in geometry (and indeed in all of mathematics and well as art and engineering) that we believe deserve to be called aesthetic experiences.

We believe that mathematics is a natural and deep part of human experience and that experiences of meaning in mathematics should be accessible to everyone. Much of mathematics is not accessible through formal approaches except to those with specialized learning. However, through the use of non-formal experience and geometric imagery, many levels of meaning in mathematics can be opened up in a way that most people can experience and find intellectually challenging and stimulating.

\footnotetext{
${ }^{1}$ Partial support for this work was provided by the National Science Foundation's National Science, Technology, Engineering, and Mathematics Education Digital Library (NSDL) Program under grant DUE-0226238.
} 
A formal proof, as we normally conceive of it, is not the goal of mathematics - it is a tool - a means to an end. The goal is to understand meanings. Without understanding, we will never be satisfied - with understanding, we want to expand the meanings and to communicate them to others.

Many formal aspects of mathematics have now been mechanized and this mechanization is widely available on personal computers or even handheld calculators, but the experience of meaning in mathematics is still a human enterprise. Experiencing meanings is vital for anyone who wishes to understand mathematics, or anyone wishing to understand something in their experience through the vehicle of mathematics. We observe in ourselves and in our students that these are, at their core, aesthetic experiences.

In this chapter we will tell some stories of our experience of meanings in geometry and art. David's story starts with art and ends with geometry, while Daina's story starts with geometry and ends with art. However the bulk in the middle we both share.

\section{David's Story: From ARt to Mathematics}

I have always loved geometry and was thinking about geometric kinds of things since I was very young as evidenced by drawing (Figure 1) that I made when I was 6 years old.

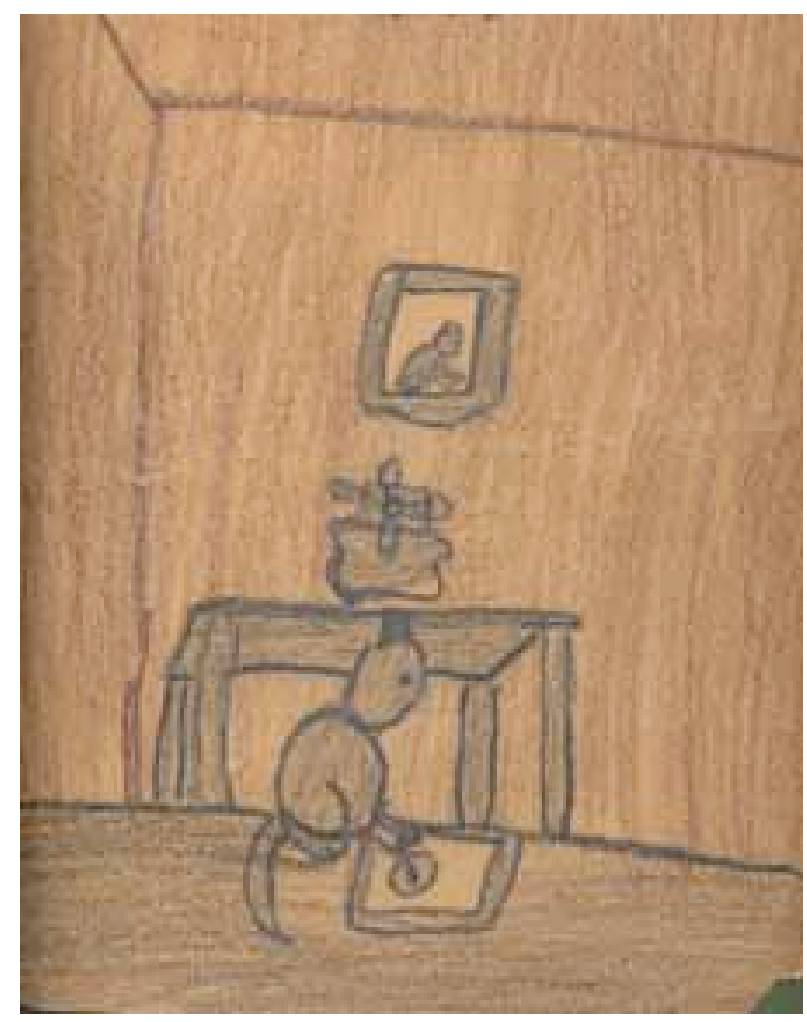

Figure 1. David's drawing (crayons on paper, $9^{\prime \prime} \times 6^{\prime \prime}$ )

Note that the drawing is of a cat drawing a picture of a cat (who is presumably drawing a picture of a cat ... Notice also the perspective from the point-of view of the cat - for example the underside of the table. I was experiencing geometry meanings.

But I did not realize that the geometry, which I experienced, was mathematics or even that it was called "geometry". I was not calling it geometry -- I was calling it drawing or design or not 
calling it anything and just doing it. I did not like mathematics in school because it seemed very dead to me -- just memorizing techniques for computing things and I was not very good at memorizing. I especially did not like my high school geometry course with its formal twocolumn proofs. But I kept doing geometry in various forms in art classes, woodcarving, carpentry, out exploring nature, or by becoming involved in photography. This continued on into the university where I was a joint physics and philosophy major and took only those mathematics courses that were required for physics majors. I became absorbed in geometry-based aspects of physics: mechanics, optics, electricity and magnetism, and relativity. On the other hand, my first mathematics research paper (on the geometry of Venn diagrams for more than 4 classes) evolved from a course on the philosophy of logic. There were no geometry courses except for analytic geometry and linear algebra, which only lightly touched on anything geometric. So, it was not until my fourth and last year at the university that I switched into mathematics because I was finally convinced that the geometry that I loved really was a part of mathematics.

Since high school, I have never taken a course in geometry because there were no geometry courses offered at the two universities that I attended. Now I am a geometer and in the mid-70's starting teaching a Euclidean geometry course. My concern that both my students and I should experience the meanings in the geometry quickly led me into conflict with the traditional formal approaches. For example:

\section{EXPeriencing "Undefined" Terms}

In geometry, "point" and "straight line" are usually labeled as "undefined terms". In a formal sense, something has to be undefined because it is impossible to define everything without being circular. However, if we want to pay attention to meanings in geometry, then we must still ask "What is the meaning of "point'?" and "What is the meaning of 'straight'?" The standard formal approach of saying they are undefined terms pushes away these questions.

What is the meaning of 'point'? Euclid has one answer - in Heath's translation of The Elements [Euclid], "A point is that which has no parts." This is one meaning of point. Point has another meaning in geometry and mathematics that can be experienced by imagining zooming in on the point. A Tibetan monk/artist/geometer explained this to one of us by saying: "Imagine a poppy seed. Now imagine in this poppy seed a temple and in the middle of the temple a Buddha and in the navel of the Buddha another poppy seed. Now in that poppy seed imagine a temple and in the temple a Buddha and in the navel of the Buddha another poppy seed. Now in that poppy seed imagine ... (and keep going) ... . Where is the point?" See Figure 2. [As we write this, we notice some similarity between this zooming and ideas in David's picture of a cat drawing a picture of a cat....]

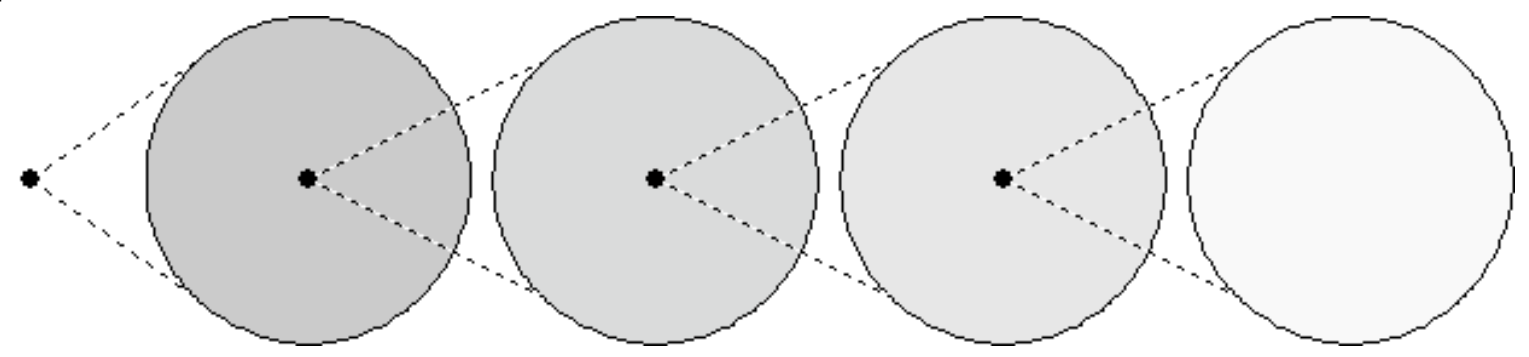

Figure 2. Zooming in on a point. Where is the point? 
These meanings of 'point' are not the same and thus bring in the question: Why and how are these meanings related? This is a why-question that often confronts calculus students when looking at the meanings of tangent, limit, and the definite integral.

What is the meaning of straight? This is the question that starts both of the geometry books ([HT-eg] and [HT-dg]) that we have written. Of course, whether a text or teacher allows this discussion or not, students (in fact, it appears, most human beings) have an experience of meanings of straight. The meanings of straight are part of the foundation for meaning in geometry.

One common meaning for 'straight' is 'shortest distance'. This meaning can be used in practice to produce a straight line by stretching a string (or rubber band). Figure 3.

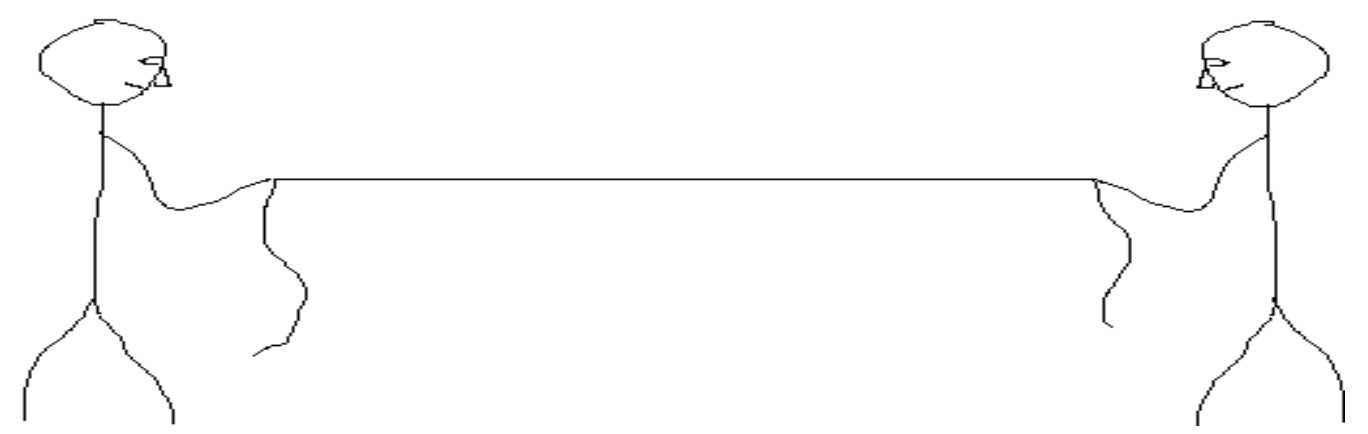

Figure 3. Straight as the shortest distance

There is another meaning in the realization that a straight line is very symmetric - "it does not turn or wiggle", "in the plane, both sides are the same". Straight lines have at every point the following symmetries: Reflection thru the line, Reflection perpendicular to the line, Half-turn about a point on the line, Translation along the line, and so forth. See Figure 4. This symmetry meaning is in line with Heath's translation of Euclid's definition of straight line as "a line that lies evenly with the points on itself", which he clarifies in a footnote: "we can safely say that the sort of idea which Euclid wished to express was that of a line ... without any irregular or unsymmetrical feature distinguishing one part or side of it from anther."

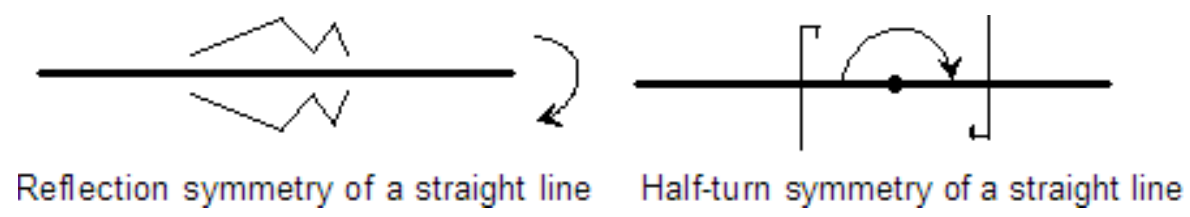

Figure 4. Straight as symmetric

Using these experiential based meanings of straightness we can ask what are the straight lines on the surface of a sphere? If we look at this question from a view outside of the sphere, then the answer is clearly that there are no straight lines on a sphere. This is the extrinsic pointof-view. On the other hand, there is an intrinsic point-of-view: Imagine yourself to be a bug crawling on a sphere. The bug's universe is the just the spherical surface. What paths on the sphere would the bug experience as straight? After some exploration, we can convince ourselves that the great circles on the sphere are the curves that have the same symmetries (with respect to the sphere) that ordinary straight lines have with respect to the plane. We thus say that the great circles are intrinsically straight. A more usual approach in texts is to define straight lines on the 
sphere to be the great circles - but, again, this blocks contact with the meaning (and thus the aesthetics).

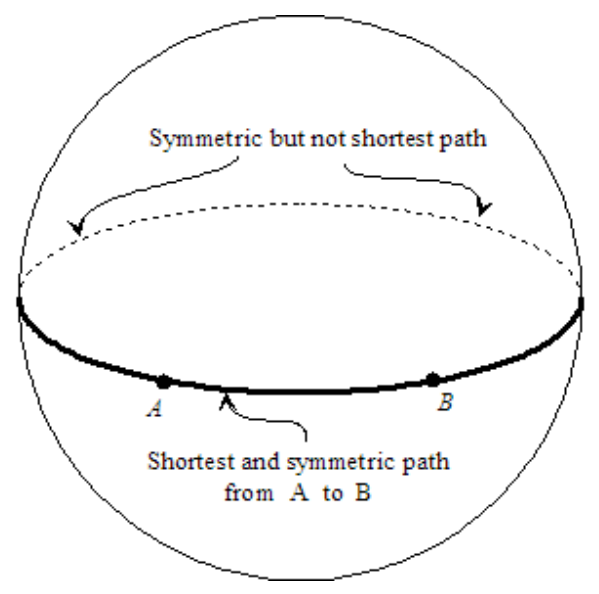

Figure 5. Intrinsically straight on a sphere

So again: Why and in what way are these two meanings ("shortest" and "symmetric") related? On the sphere we see (Figure 5) that for two nearby points of the equator (a particular great circle) the shortest distance is along the equator but that there is another straight (in the meaning of symmetric) path between the same two points that traverses the equator in the opposite direction going the long-way-around. Thus "symmetric" is not always "shortest". In addition, there are surfaces with corners (see Figure 6) in which the shortest path is not symmetric.

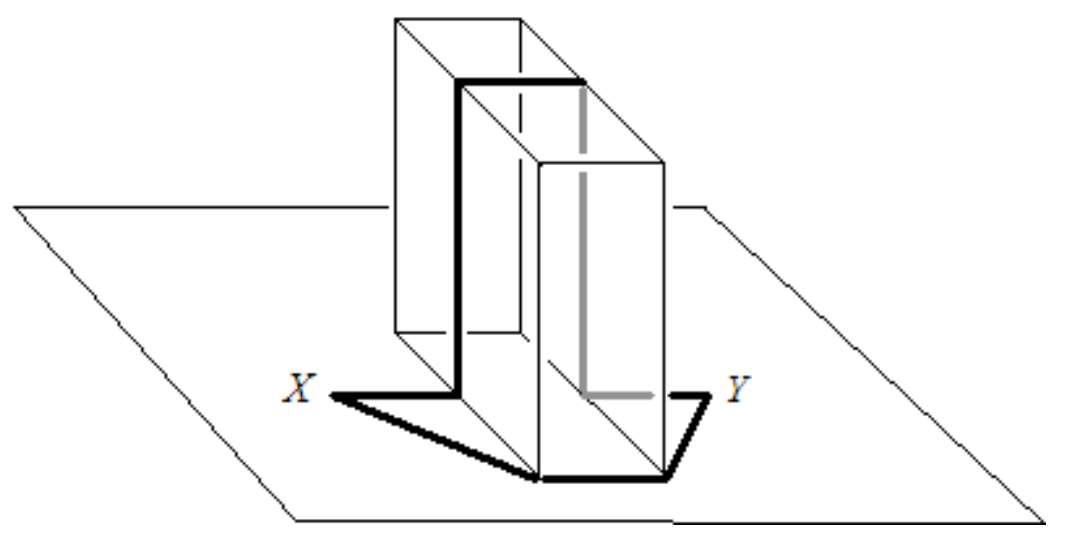

Figure 6. 'Shortest' is not the same as 'symmetric'

\section{Proofs as Convincing Communications THAT ANSWERS - WhY?}

Much of our view of the nature of mathematics is intertwined with our notion of what is a proof. This is often particularly true with geometry, which has traditionally been taught in high school in the context of "two-column" proofs. Instead we propose a view of proof as a convincing communication that answers a why-question. 
The series of books entitled Proofs Without Words [Nelson] contain many examples of visual proofs that provide an experience of why something is true - a experience that is in most cases difficult to obtain from the usual formal proofs. For example, from [Nelson] we have the following result attributed to Galileo:

Note that

We can easily check that this is true by simply adding the number. These are the cases of $n=2,3$ of the more general equation:

So the question is whether the general equation holds and, if so, why does it hold? One way to answer the first question is to apply an argument by mathematical induction, though such an argument is unlikely to satisfy the why-question. Instead look at Figure 7.

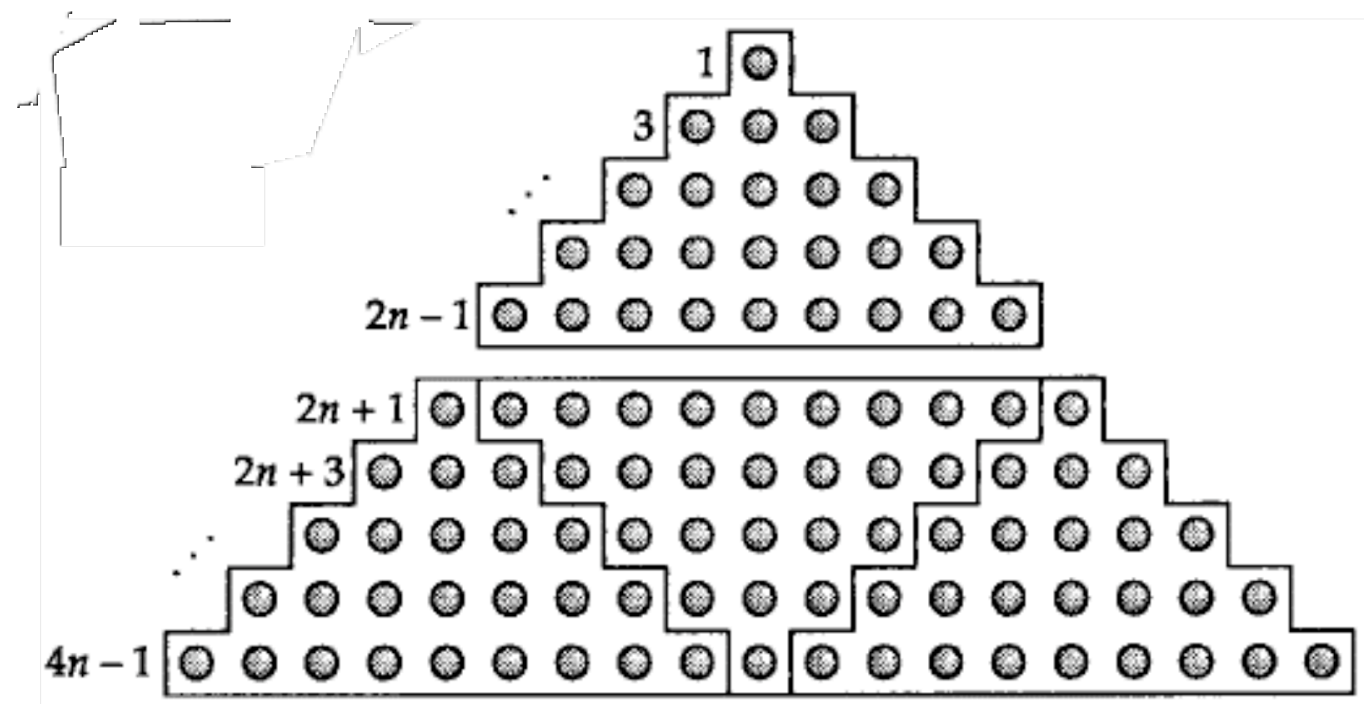

Figure 7. Proof Without Words ${ }^{2}$

Through picture, one can directly experience the meaning of Galileo's result and see both that is true and why it is true. The proof by induction would answer the question: How does Galileo's result follow from Peano's Axioms?" Most people (other than logicians) have little interest in that question.

CONCLUSION: In order for a proof to be an aesthetic experience for us, the proof must answer our why-question and relate our meanings of the concepts involved.

As further evidence toward this conclusion: You have probably had the experience of reading a proof and following each step logically but still not being satisfied because the proof did not lead you to experience the answer to your why-question. In fact most proofs in the literature are not written out in such a way that it is possible to follow each step in a logical formal way. Even if they were so written, most proofs would be too long and complicated for a

\footnotetext{
${ }^{2}$ From [Nelson], page 115. Copyright by Mathematical Association of America, 1993.
} 
person to check each step. Furthermore, even among mathematics researchers, a formal logical proof that they can follow step-by-step is often not satisfying. For example, David's research paper ["A simplicial complex whose product with any ANR is a simplicial complex," General Topology 3 (1973), pp. 81-83] has a very concise simple (half-page) proof that more questions from other mathematicians about that paper than about any of his other research papers and most of the questions were of the sort: "Why is it true?" "Where did it come from?" "How did you see it?" They accepted the proof logically but were not satisfied.

Sometimes we have legitimate why-questions even with respect to statements traditionally accepted as axioms. Another one is Side-Angle-Side (or SAS): If two triangles have two sides and the included angle of one congruent to two sides and the included angle of the other, then the triangles are congruent. You can find SAS listed in some geometry textbooks as an axiom to be assumed; in others it is listed as a theorem to be proved and in still others as a definition of the congruency of two triangles. But clearly one can ask, "Why is SAS true in the plane?" This is especially true because SAS is false for (geodesic) triangles on the sphere. So one can naturally ask, "Why is SAS true on the plane but not on the sphere?"

Let us look at another example - the Vertical Angle Theorem: If l and l' are straight lines, then the angle $\square$ is congruent to the angle $\square$. Figure 8

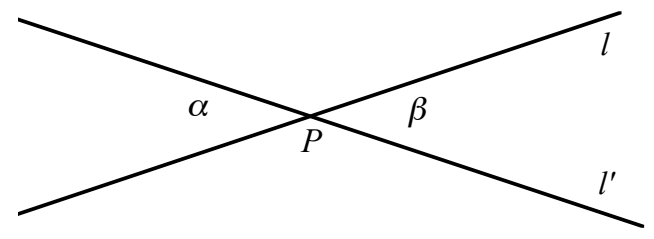

Figure 8. Vertical Angle Theorem

The traditional proof of this in high school geometry is to label the upper angle between $\square$ and $\square$ to be $\square$ and then assert $\square+\square=180^{\circ}$ and $\square+\square=180^{\circ}$. The usually proof then conclude that $\square$ is congruent to $\square$ because they are both equal to $180^{\circ}-\square$. This proof seems fine until one worries about whether the rules of arithmetic apply in this way to angles. The traditional solution in high school is to several "Ruler and Protractor" Axioms to assert the properties needed. We do not know of anyone for whom this proof with the attending axioms is aesthetic (though it may be convincing.) This proof seems to be an unnecessarily complicated answer to the question: Why are the vertical angles congruent?

For about ten years of teaching this theorem in geometry course, David was satisfied with the idea of this proof, though he managed to simplify and make more geometric the necessary assumptions contained in the "Ruler and Protractor" Axioms. But then one student suggested that the vertical angles were congruent because both lines had half-turn symmetry about their point of intersection, $P$. David's first reaction was that her argument could not possibly be a proof - it was too simple and did not involve everything in the standard proof. But she persisted patiently for several days and David's meanings deepened. Now her proof is much more convincing to him than the standard one.

Even more importantly, the meaning of the student's "half-turn" proof is closer to the meaning in the statement of the theorem. To see this look at the situation depicted in Figure 9. 


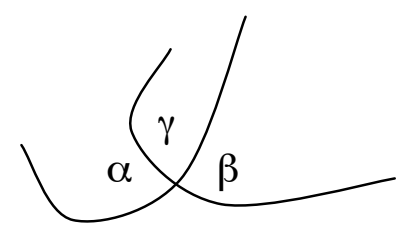

Figure 9. Are the opposite angles $\square$ and $\square$ the same?

Here there is no symmetry; however, the standard proof seems to apply and gives a misleading result. If you either zoom in on the point of intersection until that the curves are indistinguishable from straight line segments or you define the angle to be the angle between the lines tangent to the curves at the intersection, then symmetry applies and the angles $\square$ and $\square$ are congruent. However the standard proof does provide a way to discuss this except through a discussion of when the ruler and protractor axioms are valid.

You may ask, "But, at least in plane geometry, isn't an angle an angle? Don't we all agree on what an angle is?" Well, yes and no. Consider the acute angle depicted in Figure 10.

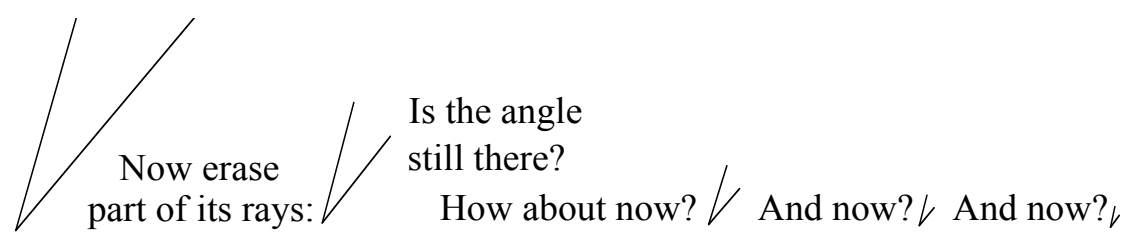

Figure 10. Where is the angle?

The angle is somehow at the corner, yet it is difficult to express this formally (note that the zooming meaning of point seems to be involved here). As evidence of this difficulty, we have looked in all the plane geometry books in Cornell's mathematics library and found their definitions for "angle." We found nine different definitions! Each expressed a different meaning or aspect of "angle" and thus, each would potentially lead to a different proof of any theorem that crucially involves the meaning of angle.

\section{Experiencing the Hyperbolic Plane}

Starting soon after the Elements were written and continuing for the next 2000 years mathematicians attempted to either prove Euclid's Fifth Postulate as a theorem (based on the other postulates) or to modify it in various ways. These attempts culminated around 1825, when Nicolai Lobatchevsky (Russian, 1792-1850) and János Bolyai (Hungarian, 1802-60) independently discovered a geometry that satisfies all of Euclid's Postulates and Common Notions except that the Fifth (Parallel) Postulate does not hold. It is this geometry that is called hyperbolic geometry. The first description of hyperbolic geometry was given in the context of Euclid's Postulates, and it was proved that all hyperbolic geometries are the same except for scale (in the same sense that all spheres are the same except for scale).

In the 19th century mathematicians developed three so-called "models" of hyperbolic geometry. In 1869-71, E. Beltrami (Italian, 1835-1906) and F. Klein (German, 1849-1925) developed the first complete model of hyperbolic geometry (and first called the geometry "hyperbolic"). In the Beltrami-Klein Model the hyperbolic plane represented by the interior of a 
circle, and the straight lines are represented by the (straight) chords of that circle, and its "reflection" about a chord is a projective transformation that take the circle to itself and leave the chord pointwise fixed. Around 1880, H. Poincaré developed two related models. In the Poincaré Disk Model the hyperbolic plane is represented by the interior of a circle with the straight lines being circular arcs perpendicular to this circle. In the Poincaré Upper Half Plane Model the hyperbolic plane is represented by half a plane on one side of a line with the straight lines being semicircles that are perpendicular to that line. All three models distort distances (in ways that are analytically describable); but the Beltrami-Klein Model represents hyperbolic straight lines as Euclidean straight-line segments and both Poincaré models accurately represent angles. For more details on these hyperbolic models see [HT-eg, Chapter 16].

These models of hyperbolic geometry have a definite aesthetic appeal, especially through the great variety of repeating patterns that are possible in the hyperbolic plane. The artist M. C. Escher used patterns based on these hyperbolic models is several well-known prints, for example in Figure 11.

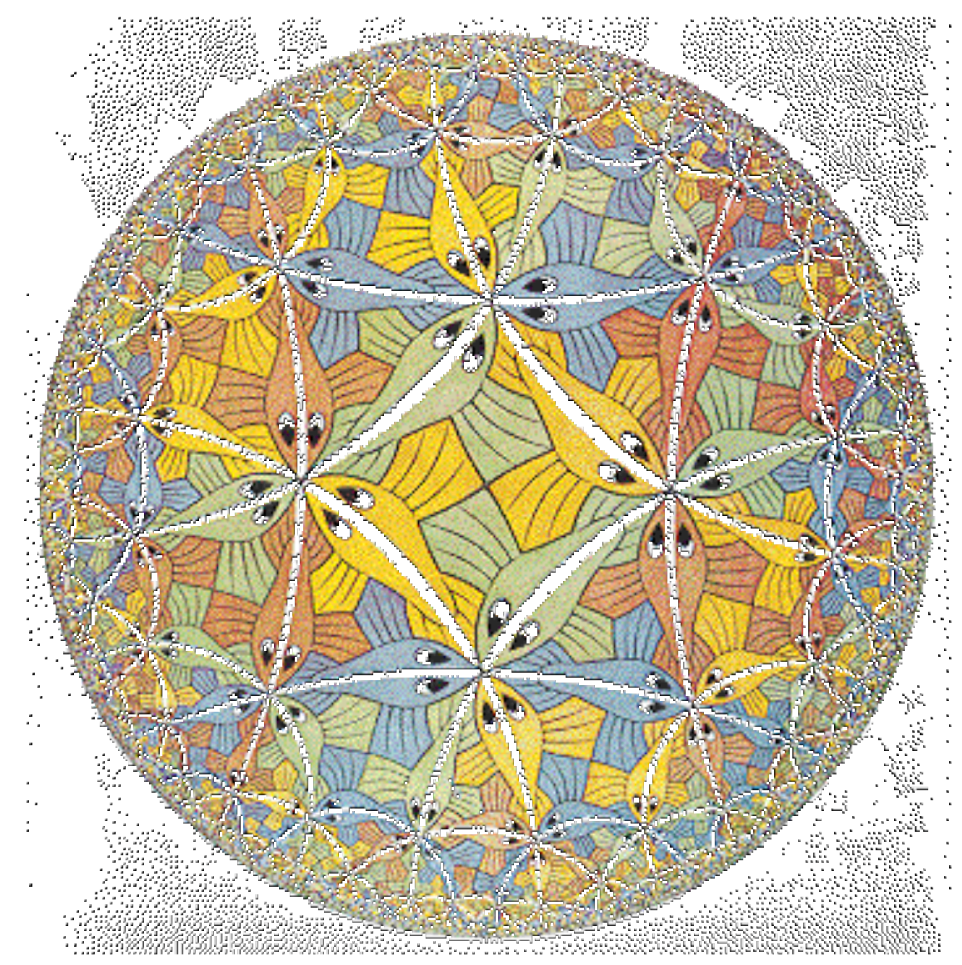

Figure 11. Escher's Circle Limit III, based on the Poincaré Disk model.

For the past $125+$ years these models have been very useful for mathematically studying hyperbolic geometry. However, many students and mathematicians (including the two of us) have desired a more direct experience of hyperbolic geometry - an experience similar to experiencing spherical geometry by handling a physical sphere. In other words, the experience of hyperbolic geometry assessable through the models did not directly include an experience of the intrinsic nature of hyperbolic geometry.

Mathematicians looked for surfaces that would be the complete hyperbolic geometry in the same sense that a sphere has the complete spherical geometry. In 1868, Beltrami described a surface, called the pseudosphere (Figure 12), which locally has hyperbolic geometry. The pseudosphere also has certain aesthetic appeals. 


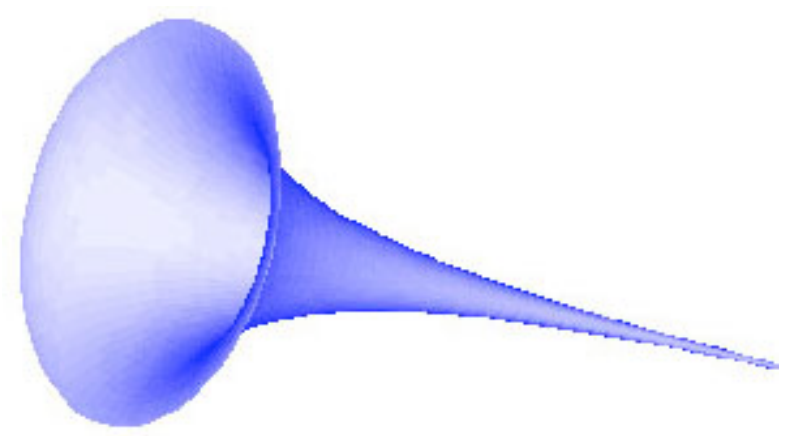

Figure 12. Pseudosphere ${ }^{3}$

However, the pseudosphere allows only a very limited experience of hyperbolic geometry because any patch on the surface that wraps around the surface or extend to the circle boundary does not have the geometry of any piece of the hyperbolic plane. In 1901, David Hilbert [Hilbertb] proved that it is impossible to use real analytic equations to define a complete surface whose intrinsic geometry is the hyperbolic plane. In those days "surface" normally meant one defined by real analytic equations and so the search for a complete hyperbolic surface was abandoned and still today many texts state incorrectly that a complete hyperbolic surface is impossible. In 1964, N. V. Efimov [Efimov] extended Hilbert's result by proving that there is no isometric embedding defined by functions whose first and second derivatives are continuous. However, in 1955, Nicolas Kuiper [Kuiper] proved, without giving an explicit construction, the existence of complete hyperbolic surfaces defined by continuously differentiable functions. Then, in the 1970 's, William Thurston described the construction of a surface (that can be made out of identical paper annuli) that closely approximates a complete hyperbolic surface, see [Thurston], pages 49 and 50, and Figure 13. The actual hyperbolic plane is obtained by letting the width of the annular strips go to zero.

\footnotetext{
${ }^{3}$ Drawn by SGeometry is a Power Macintosh program written by Andrea Mantler http://bkocay.cs.umanitoba.ca/Students/Pseudosphere.html
} 


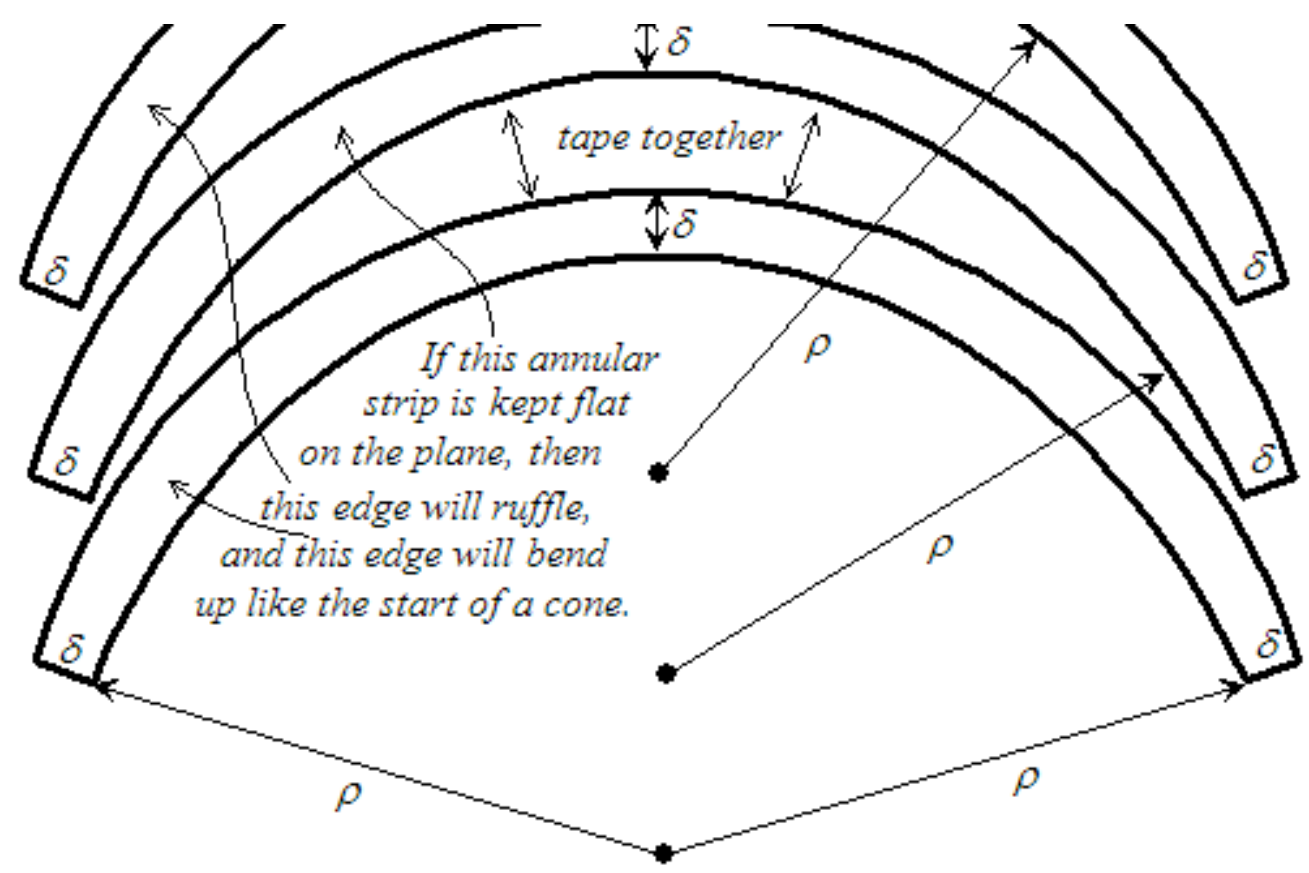

Figure 13. Construction of the annular hyperbolic plane

Daina figured out in 1997 how to crochet the hyperbolic plane following Thurston's annular construction idea. See Figure 14.

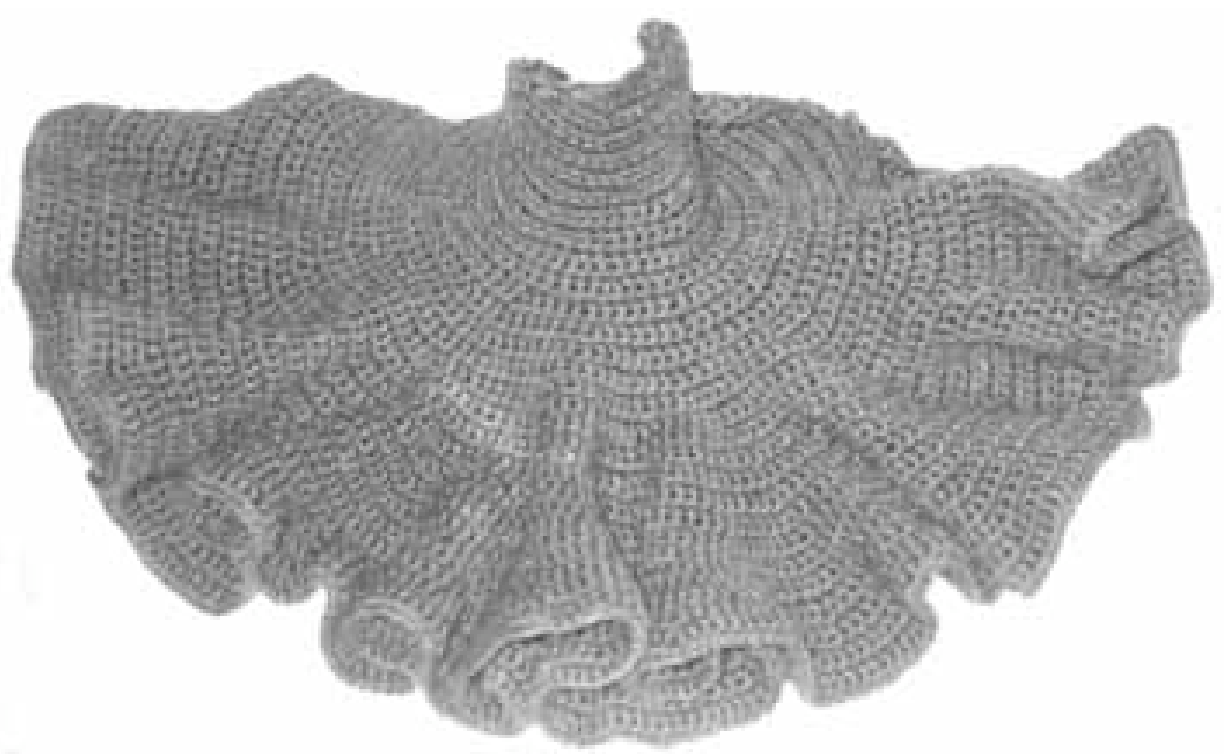

Figure 14. Crocheted hyperbolic plane $e^{4}$

Directions for constructing Thurston's surface out of paper or by crocheting can be found in [HT-eg] or [HT-croc]. In these references there is also a description of an easily constructible polyhedral hyperbolic surface, called the "hyperbolic soccer ball", that consists of heptagons each surrounded by 7 hexagons (the usual spherical soccer ball consist of pentagons each surrounded by 5 hexagons). This polyhedral surface was discovered by Keith Henderson,

\footnotetext{
${ }^{4}$ Model crocheted by Daina Taimina. Photograph by David W. Henderson
} 
David's son, and is a very accurate polyhedral approximation to the hyperbolic plane. See Figure 15.

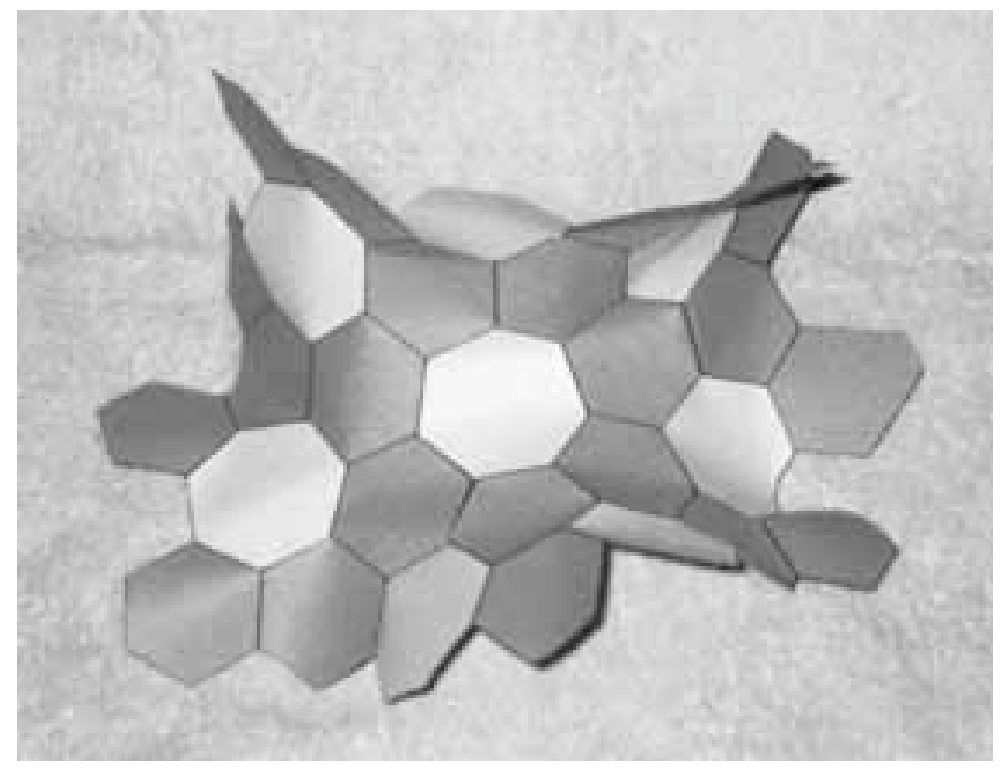

Figure 15. Hyperbolic soccer ball ${ }^{5}$

The geodesics ("intrinsic straight lines") on a hyperbolic surface can be found using the symmetry meaning of straightness discussed above. For example, the geodesics can be found by folding the surface (in the same way that folding a sheet of paper will produce a straight line on the paper). This folding also determines a reflection about the geodesic.

Now, with these surfaces, we can have a more direct experience of the meanings in hyperbolic geometry. And, very importantly, we can experience the connections between these meanings and the three models discussed above. These models can now be interpreted as projections (or maps) onto a region in the plane of the hyperbolic surface that distorts it in a similar way that projections (maps) of a sphere (such as the Earth) distort distances and areas and/or angles. This is important because these models are used to study the details of hyperbolic geometry and the surface allows us direct experience with the intrinsic geometry.

Before we had experiences with the surface all experience of hyperbolic was formal study through axioms systems and through analytic study of the models. The models provided aesthetics experiences, but this was not connected with the geometric meanings. For example, the question that we (and most students) had was: Why geodesics in the Poincaré models are represented by semicircles? To us, the models were more like artistic abstractions. But, after constructing the surfaces we could see how and why the geodesics are represented in the way they are. See [HT-eg] or [HT-croc] for more details of these connections, including proofs that the intrinsic geometry of the surfaces is the same geometry represented by the models.

Radius and curvature of the hyperbolic plane: Since all hyperbolic planes are the same up to scale, most treatments of the hyperbolic plane consider the curvature to be $\square 1$. It is very difficult to give meaning to the effects of the change of curvature without looking at actual physical hyperbolic surfaces with different curvatures.

\footnotetext{
${ }^{5}$ Photo and model by Keith Henderson, used here by permission.
} 


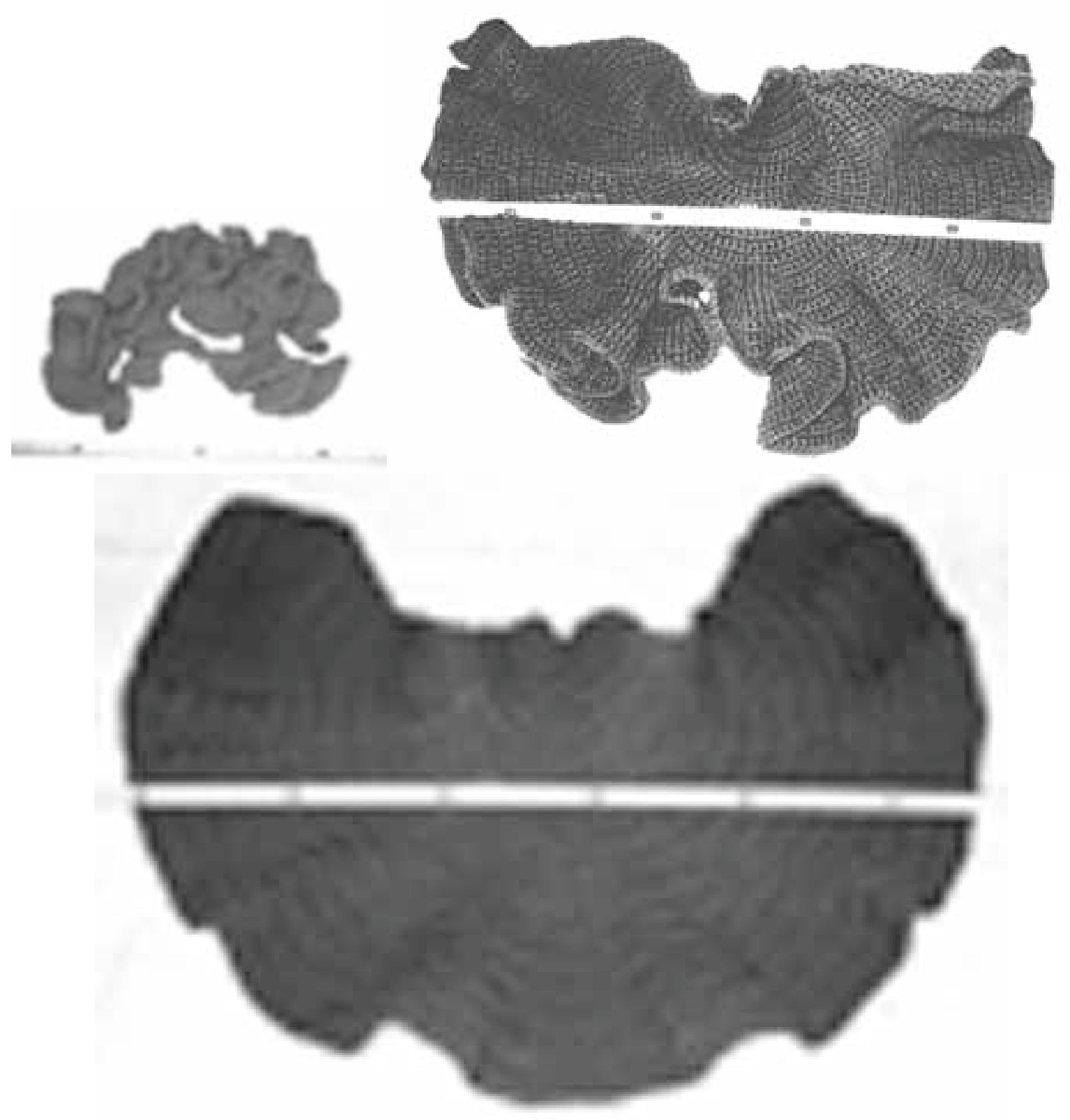

Figure 16. Hyperbolic planes with different radii ${ }^{6}$

Each sphere has a radius, $r$, (which is extrinsic to the sphere) and its (Gaussian) curvature (as defined in differential geometry) is $1 / r^{2}$. In a similar way, each hyperbolic plane has a radius, $r$, which turns out to be the (extrinsic) radius of the annuli that go into its construction and the (Gaussian) curvature of the hyperbolic plane is $\square 1 / r^{2}$. We were not aware of any meaning for the radius of a hyperbolic plane before our experiences with these surfaces. From a theoretical perspective changing the radius or curvature is merely a change of scale and spheres of radii

\footnotetext{
${ }^{6}$ Crocheted by Daina Taimina. Photographs by David W. Henderson.
} 
$4 \mathrm{~cm}, 8 \mathrm{~cm}$, and $16 \mathrm{~cm}$ look very much alike. However, we were shocked when we looked at the hyperbolic planes with these same radii (Figure 16). There is felt difference that is not present in the spheres of the same radii (the main reason for this difference seems come from the fact of exponential growth in the hyperbolic plane). This experience of the meaning of radius of a hyperbolic plane was profound (and aesthetic) to us. We are forced by these experiences to look deeper mathematically into the meaning of radius and curvature and local and global natures of the hyperbolic plane.

Ideal triangles: By exploring the possible shapes of large triangles on the hyperbolic surface (see Figure 17) we see that they seem to become more and more the same shape as they become large. This leads to the theorem (proved by using the models) that all ideal triangles (with vertices at infinity) are congruent and have area equal $\square r^{2}$ ! (Note this is the same as the extrinsic area of the circles determined by the annuli in the construction.)

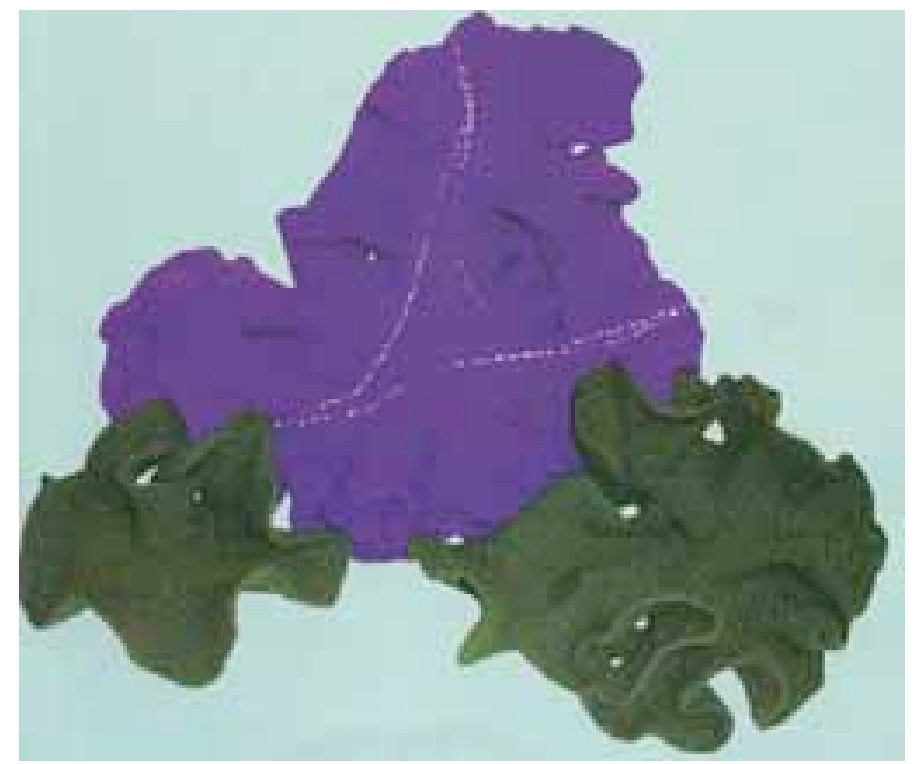

Figure 17. Ideal triangle on the hyperbolic plane ${ }^{7}$

Horocycles or Horocircles: By experiencing the annular construction (Figure 14) it is easy to see that the curves perpendicular to the annuli (that is, curves that run is the radial direction) posses reflection symmetry and thus are geodesics. In addition, they are asymptotic to each other at infinity. Most treatments of hyperbolic geometry define horocycles as those curves that are orthogonal to a collection of asymptotic geodesics. Thus the annuli (in the limit as their width goes to zero) are horocycles. Both of us had studied hyperbolic geometry and its models; but exploring the hyperbolic surface was the first time we had experienced the horocycles in way that made clear their connections with the curvature and how, as many books say, they can be described as circles with infinite (intrinsic radii).

\section{Experiencing Geometry in Machines}

Recently, we have been working on an NSF project to examine the mathematics inherent in a collection of $19^{\text {th }}$ Century mechanisms and the inclusion of these mechanisms and commentaries

\footnotetext{
${ }^{7}$ Crocheted and photographed by Daina Taimina.
} 
and learning modules as part of the new NSDL (National Science Digital Library). Our experiences with these mechanisms are giving us different perspectives on geometry perspectives that arise from motion. For example, this brought us back to the question: What is straight?

When using a compass to draw a circle, we are not starting with a figure we accept as circular; instead we are using a fundamental property of circles that the points on a circle are at a fixed distance from the center. In other words we are using the definition of a circle. Is there a tool (serving the role of a compass) that will draw a straight line? If, in this case, we want to use Euclid's definition (A straight line is a line which lies evenly with the points on itself) -- it won't be of much help. One can say: We can use a straightedge for constructing a straight line! Well, how do you know that your straightedge is straight? How do you know that something is straight? How can you check that something is straight? This question was important for James Watt when he was thinking about improving steam engines - he needed a mechanism to convert circular motion into straight-line motion and vice versa. In 1784 Watt found a solution (which he called "parallel motion") that consisted of a linkage with 6 links. His "parallel motion" was a practical (and aesthetic) solution to his problem, but it produced only approximate straight-line motion - it actually produces a stretched out figure eight. Mathematicians were not satisfied with the approximate solution and worked for almost a hundred years to find exact solutions of the problem. A linkage (Figure 18) that draws an exact straight line was first reported by Peaucellier in 1864. (See [HT-MAA] for a discussion of the relevant history.)
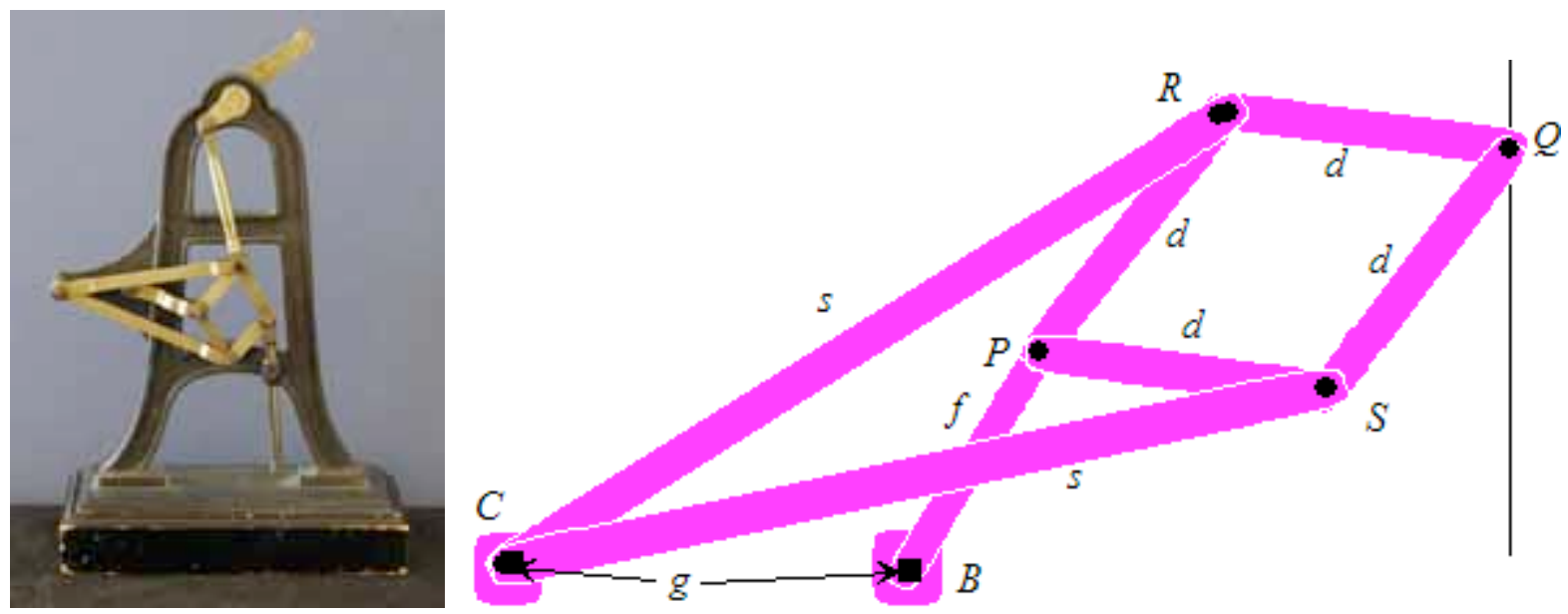

Figure 18. Peaucellier linkage ${ }^{8}$

Why does the Peaucellier linkage draw a straight line? We suggest the reader connect to a website where this linkage is depicted in motion. (For example, see http://KMODDL.library.cornell.edu/.) One can derive, as an exercise in analytic geometry, that the point $Q$ will always lie along a straight line - but this does not answer the why-question. Especially difficult is to see any relationship with either of the 'shortest' or 'symmetry' notions of straightness - Is there perhaps a different meaning of straightness that is operative here?

In the "inversor" (the links joining $C, R, Q, S, P$ in Figure 18) the points $P$ and $Q$ are inverse pairs with respect to a circle with center $C$ and radius $r=\sqrt{ }$-- analytically, this means that $\mathrm{d}(C, P) \mathrm{d}(C, Q)=r^{2}$. Here, the crucial property of circle inversion is that it takes circles to circles.

\footnotetext{
${ }^{8}$ Photograph copyright by Francis C. Moon. Used here by permission.
} 
(For details on circle inversion, see [HT-EG] Chapter 14.) After experiencing the motion of the linkage we see that $P$ is constrained (by its link to the stationary $B$ ) to travel in a circle around $B$ and thus $Q$ must be traveling along the arc of a circle - the radius and center of this circle is varied by changing the position of the fixed point $B$ the length of the link $B P$. Thus, the Peaucellier linkage draws (at $Q$ ) the arc of a circle without using the center of that circle. If the lengths $C B$ and $B P$ are equal then the circle on which $P$ moves goes through the center $C$; and, since points near $C$ are inverted to points near infinity, the circle that $Q$ lies on must go through infinity! How can a circle go through infinity? Answer: Only if the circle has infinite radius. $A$ circle with infinite radius (and thus zero curvature) is a straight line. We now have a third meaning for straight line; and the Peaucellier linkage is a tool for drawing a straight line using this meaning.

Notice that in the previous section on hyperbolic geometry we pointed out the horocycles in the hyperbolic plane can be seen as circles of infinite radius. Thus circles with infinite radius are not straight on the hyperbolic plane, even though they are straight on the Euclidean plane. This proves that 'straight' as 'circle of infinite radius' is a different meaning than either 'straight' as 'symmetric' or 'straight' as 'shortest'.

Behind this discussion is the theory of circle inversions, which are one of the most aesthetic geometric transformations and is often used in modern art. (See, for example, Figure 19.) But the experience of the linkage as a mechanism that draws a circle without using its center allows one to then understand how the linkage can draw a circle of infinite radius and thus a straight line.

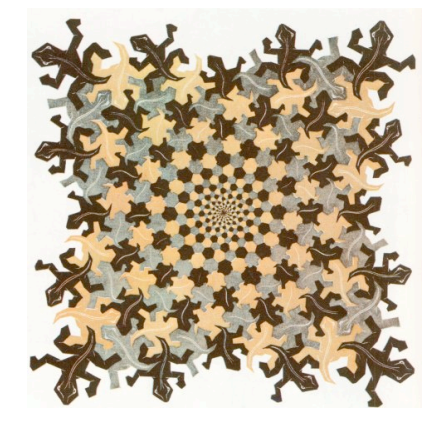

Figure 19. Inversion-based art

Peaucellier's linkage is one of 39 straight-line mechanisms in Cornell University's collection, which has more then 220 kinematic models designed by Franz Reuleaux (1829-1905). Those models are a rediscovery of lost $19^{\text {th }}$ century machine design knowledge. Franz Reuleaux is often called a "father of modern machine design". Reuleaux's two most important books contain hundreds of drawings of machines and mechanisms. To compliment his books, Reuleaux designed and built over 800 kinematic models to illustrate his theory of machines. The models in the Cornell collection clearly show the aesthetic style of Reuleaux. (To read more about Reuleaux, his mechanisms, and his theory of machines, see the article [Moon] which also contains many further references.)

As we have been exploring the mathematics behind the Reuleaux models for the NSDL project, we are surprised how much aesthetics we can find there -- not only in machine design, but also in mathematics. These experiences caused us to ask about the relationships between mathematics, engineering, and art. Leonardo da Vinci is a well know example of this interrelationship; but we found that there seems to be a broader connection. For example, 
Reuleaux in his Kinematics [Reuleaux] refers specifically to the artist and to experiences of deeper meanings similar to our discussion at the beginning of this chapter:

He who best understands the machine, who is best acquainted with its essential nature, will be able to accomplish the most by its means. (p. 2)

In each new region of intellectual creation the inventor works as does the artist. His genius steps lightly over the airy masonry of reasoning which it has thrown across to the new stand point. It is useless to demand from either artist or inventor an account of his steps. (p. 6)

The real cause of the insufficiency of [previous classification systems] is not, however, the classification itself; it must be looked for deeper. It lies ... in the circumstance that the investigations have never been carried back far enough,--back to the rise of the ideas; that classification has been attempted without any real comprehension being obtained of the subjects to be classified. (p. 18)

In addition, the History of Engineering, Eugene Ferguson wrote [Ferguson]:

Both the engineer and the artist start with a blank page. Each will transfer to it the vision in his mind's eye. The choice made by artists as they construct their pictures may appear to be quite arbitrary, but those choices are guided by the goal of transmitting their visions, complete with insights and meaning, to other minds. ... The engineers' goal of producing a drawing of a device -a machine or structure or system -- may seem to rule out most if not all arbitrary choices. Yet engineering design is surprisingly open-ended. A goal may be reached by many, many different paths, some of which are better than others but none of which is in all respects the one best way... (p. 23)

Ferguson also mentions that Robert Fulton (of steamboat fame) and Samuel Morse (inventor of the electrical telegraph) were both professional artists before they turned to careers in technology.

We have already mentioned the Peaucellier linkage. Another example is Reuleaux triangles, which are the most well-known of curves with constant width. If a closed convex curve is placed between two parallel lines and the lines are moved together until they touch the curve, the distance between the parallel lines is the curve's "width" in one direction. Because a circle has the same width in all directions, it can be rotated between two parallel lines without altering the distance between the lines.

The simplest non-circular constant-width curve is named the Reuleaux triangle. Mathematicians knew it earlier, but Reuleaux [Reu, p.131-146] was the first to study various motions determined by constant-width figures. A Reuleaux triangle can be constructed starting with an equilateral triangle of side $s$ and then replacing each side by a circular arc with the other two sides as radii, as in Figure 19. The resulting figure bounded by these three arcs is the Reuleaux Triangle. Its constant-width is equal to $s$, the side of original triangle. 


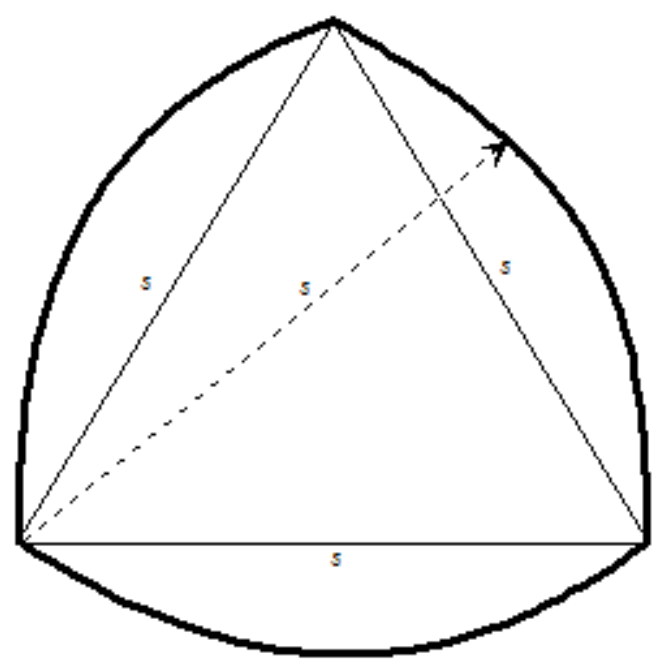

Figure 20. Reuleaux triangle

In Reuleaux's collection we find several applications of this triangle and other constantwidth curves, see Figure 21.

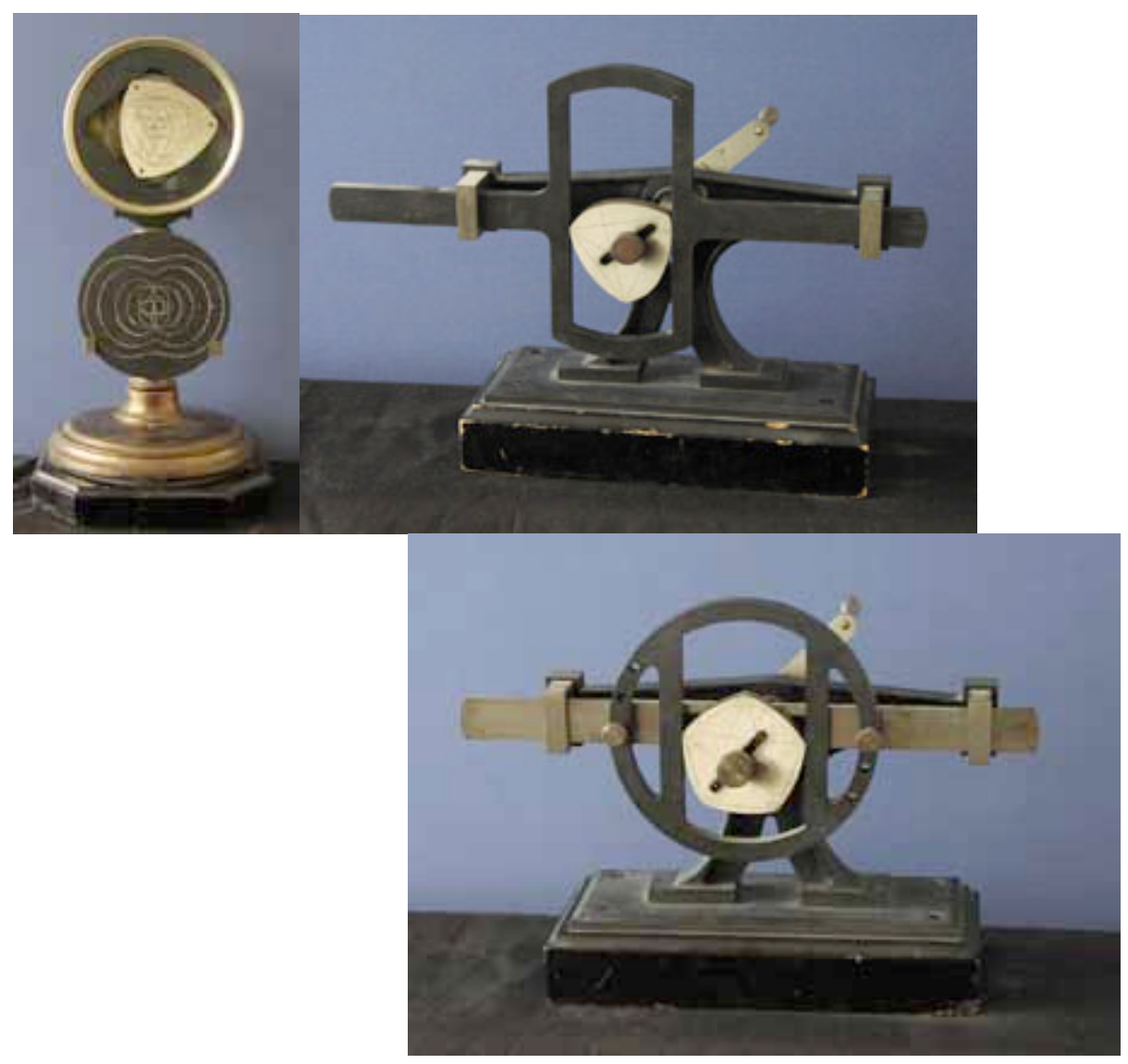


Figure 21. Some Reuleaux mechanisms using constant-width curves. ${ }^{9}$

The Reuleaux triangle fits inside a square of side $s$ and can be rotated 360 degrees within the square - this is the idea behind the drill bits that drill a (almost) square hole. And conversely, the square can rotate around the stationery Reuleaux triangle. Reuleaux did not give analytical descriptions of these motions; instead he produced many drawings, which in an aesthetically visual way show different paths of points during the motions. Reuleaux was the first one to properly describe the properties of these motions and in his model collection we can find several applications.

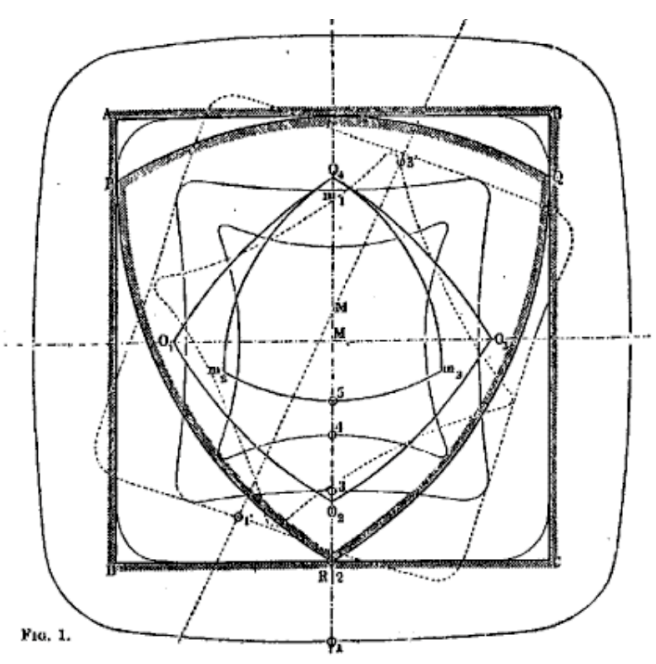

Figure 22. Reuleaux triangle moving in a square

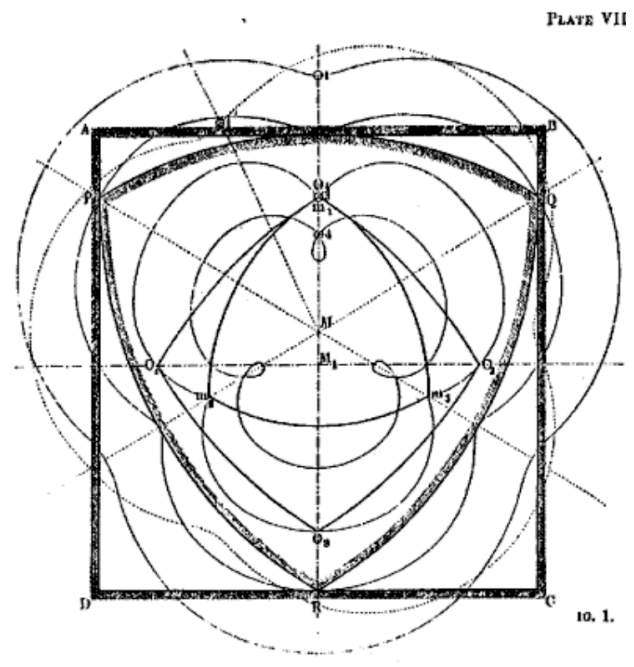

Figure 23. Square moving around a Reuleaux triangle From [Reuleaux].

Reuleaux geometrically proved a theorem that any relative motion between two shapes, $S$ and $R$, in the plane can be realized the motion of two other shapes, $c S$ and $c R$, rolling on each other with $c S$ fixed to $S$ and $c R$ attached to $R$. The rolling shapes he called centrodes, or the locus of instantaneous centers. Figures 22 and 23, show the centrodes $\left(\mathrm{O}_{1} \mathrm{O}_{2} \mathrm{O}_{3} \mathrm{O}_{4}\right.$ and $\left.m_{1} m_{2} m_{3}\right)$ for the relative motion of the square and the Reuleaux triangle. Since the relative motions are the same in the two figures the centrodes are necessarily the same. But the real meaning of this rolling motion can be experienced only by actually looking at the models in motion.

We give one more example of the aesthetics of the mathematics and machine connecting in Figure 24, which is a pump in Reuleaux's collection with two moving spirals - this design was actual used as a water meter. You will have to go to the NSDL website http://KMODDL.library.cornell.edu/ to properly experience this motion - our reaction at first seeing the static picture was that it could not possible work to pump anything!

\footnotetext{
${ }^{9}$ Photographs, copyright by Francis C. Moon. Used here by permission.
} 


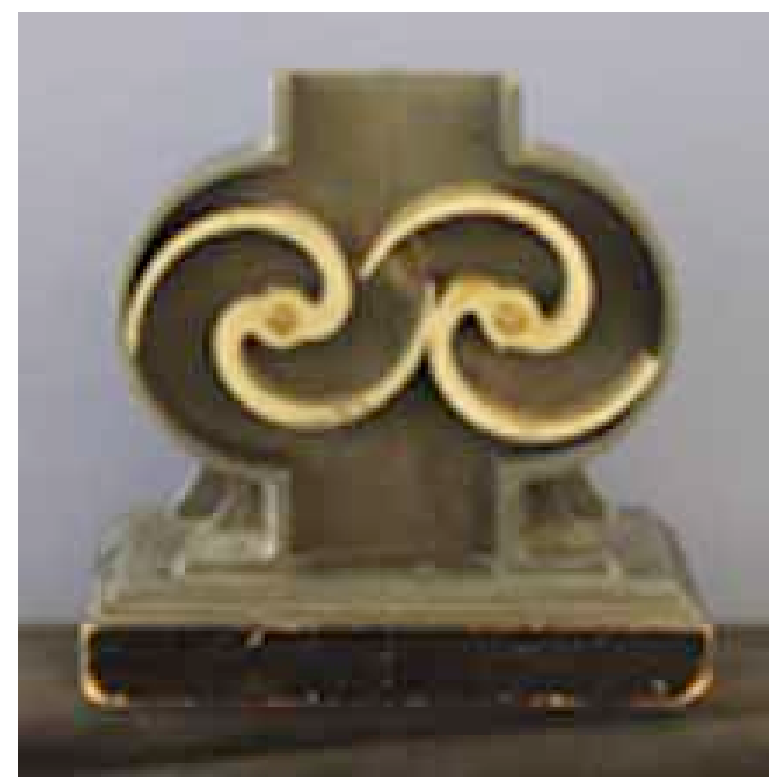

Figure 24. Spiral pump ${ }^{10}$

We have the privilege of direct physical access to these models at Cornell, but soon the whole Reuleaux kinematic model collection will be available to everybody on the web. It has been digitized and will be stored as a part of NSDL at http://KMODDL.library.cornell.edu/. The website contains videos of the motions and interactive features that will allow the virtual experience of the mechanisms in motion.

\section{Daina's Story: From Geometry to Art}

I had a lot of geometry in school and at the university. But I had only very few art lessons in school and from them I got the impression that I can not draw and that I am generally no good in art. But I liked geometry exactly for its aesthetic values -- my mathematics teachers always paid a lot of attention how we draw geometric diagrams. In the university, besides other traditional geometry courses, I also had a course in descriptive geometry and a short course on how to draw 3D geometric diagrams - both of these courses were a lot about perspective. I always loved viewing art and was interested in art history. When I was teaching the history of mathematics I was particularly interested in history of geometry and was very happy to find so many connections between geometry and art. I was fascinated with the Golden Ratio, the story of projective geometry arising from painter's perspective to a mathematical subject, and mathematics impact on art in the $20^{\text {th }}$ century (for example, cubism and later M.C. Escher). I was also teaching a course "Psychology of mathematical thinking" and it led me to wonder about creative thinking generally.

I have had many students in my mathematics classes telling me that they are taking a class just to fulfill distribution requirement but they are no good in mathematics because they are artists (poets, musicians, actors, painters) and their thinking is different. This made me wonder: Is creative thinking really different in its very essence? So I decided as an experiment to take a watercolor class knowing that I am no good in art and never was. I just wanted to get a glimpse of emotions you are going through as a student in a subject for which you know you have no

\footnotetext{
${ }^{10}$ Photograph copyright by Francis C. Moon. Used here by permission.
} 
talent. I started the watercolor class not really understanding what techniques I should use for my brush, how to mix colors, and any other such technical details. But then I realized: It was only the techniques I did not know. I had had enough aesthetics experiences in my geometry studies to have a feel for the aesthetics in painting. My geometry experiences related directly to composition and perspective in painting.

This is my painting that I did after attending only 8 watercolor classes. I started it in class and later the same day finished it at home because I could not stop. When it was dry, I looked at it and could not believe that it was me who painted it.

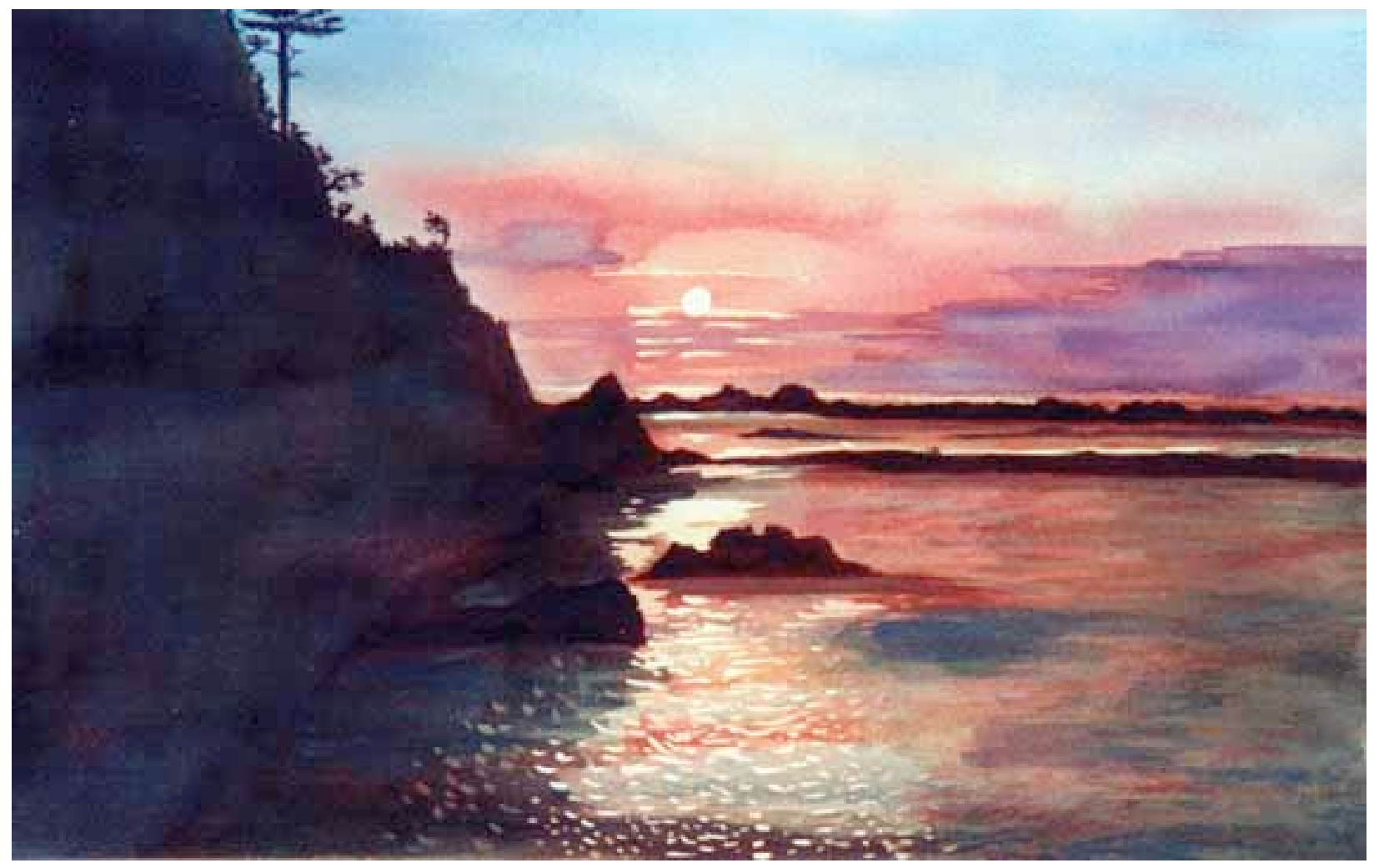

Figure 25. Daina's watercolor painting ("Sunset on Oregon Coast", $\left.27.5^{\prime \prime} \times 17.5^{\prime \prime}\right)^{11}$

\section{REFERENCES}

[Efimov] N. V. Efimov, "Generation of singularities on surfaces of negative curvature [Russian]”, Mat. Sb. (N.S.) 64 (106), pp. 286-320, 1964.

[Euclid] Euclid, Elements, T.L. Heath, ed., New York: Dover, 1956.

[Ferguson] Eugene S. Ferguson, Engineering and the Mind's Eye, MIT Press, Cambridge MA, 1992

[HT-croc] David W. Henderson and Daina Taimina, "Crocheting the Hyperbolic Plane", Mathematical Intelligencer, Vol. 23, No. 2, pp. 17-28, Spring 2001.

[HT-dg] David W. Henderson (with Daina Taimina), Differential Geometry: A Geometric Introduction, Upper Saddle River, NJ: Prentice Hall, 1998.

\footnotetext{
${ }^{11}$ Painting and photograph by Daina Taimina.
} 
[HT-eg] David W. Henderson (with Daina Taimina), Experiencng Geometry: in Euclidean, Spherical, and Hyperbolic Spaces, Upper Saddle River, NJ: Prentice Hall, 1998.

[HT-maa] David W. Henderson and Daina Taimina, "How to Use History to Clarify Common Confusions in Geometry", Chapter 1 in Using Recent History, MAA Notes, forthcoming.

[Hilbert] David Hilbert and S. Cohn-Vossen, Geometry and the Imagination, New York: Chelsea Publishing Co., 1983.

[Hilbert-b] David Hilbert, “Über Flächen von konstanter gausscher Krümmung”, Transactions of the A.M.S., 1901, pp. 87-99.

[Kemp] Martin Kemp, Visualizations: The Nature Book of Art and Science, Oxford University Press, 2000

[Kuiper] Nicolas Kuiper, "On $\mathrm{C}^{1}$-isometric embeddings, ii," Nederl. Akad. Wetensch. Proc. Ser. $A$, pp. 683-89, 1955.

[Moon] Francis C. Moon, "Franz Reuleaux: Contributions to 19th century kinematics and theory of machines", Appl Mech Rev, vol56, no. 2, March 2003, p. 261-285.

[Nelson] Roger B. Nelson, Proofs Without Words: Exercises in Visual Thinking, Washington, DC: MAA, 1993.

[Plotinus] Plotinus, The Enneads, Stephen McKenna, trans., Burdette, NY: Larson, 1992.

[Reuleaux] Franz Reuleaux, Kinematics of machinery: Outlines of a Theory of Machines, MacMillan and Co, London, 1876

[Spencer] Joel Spencer, “Opinion”, Notices of the AMS, February 2001, vol. 48, No.2, p. 165

[Thurston] William Thurston, Three-Dimensional Geometry and Topology, Vol. 1, Princeton, NJ: Princeton University Press, 1997. 